\title{
COMPUTER MODELLING OF STRUCTURES WITH ACCOUNT OF THE CONSTRUCTION STAGES AND THE TIME DEPENDENT MATERIAL PROPERTIES
}

\section{NUMERIČKO MODELIRANJE KONSTRUKCIJA UZ UVOĐENJE FAZA GRAĐENJA I SVOJSTAVA MATERIJALA ZAVISNIH OD VREMENA}

\author{
Alexander TRAYKOV \\ Raina BOIADJIEVA
}

\author{
PRETHODNO SAOPŠTENJE \\ PRELIMINARY REPORT \\ UDK: $624.01: 519.711$ \\ doi: $10.5937 / g r m k 1503029 T$
}

\section{GENERAL}

The usual way engineers calculate and design structures is assuming that they are completely built and loaded by the loads defined by the codes, including the live loads. The way and the sequence the structures are constructed is not taken into account in the design with few exceptions.

Those exceptions are some types of bridges, some assembled structures and structures with complex shapes. There are only some very general recommendations about those issues written in the codes. For the rest of the structures taking into account the influence of the sequence of construction is carried out upon designer's decision. An "ordinary" plane frame is considered in the present article. The analysis that is carried out reveals that even in an "ordinary" structure there are elements which are sensitive to the construction sequence and the construction stages shall be taken into account for their design.

Associate Professor Alexander Traykov, MSc, PhD, University of Architecture, Civil Engineering and Geodesy, Department of Structural Mechanics, 1 Hristo Smirnenski Blvd, Sofia, Bulgaria, e-mail: traykovalexander@gmail.com Raina Boiadjieva, MSc, Civil/Structural Engineer, University of Architecture, Civil Engineering and Geodesy, Department of Structural Mechanics, 1 Hris to Smirnenski Blvd, Sofia, Bulgaria, e-mail: r_boiadjieva@abv.bg

\section{DESCRIPTION OF THE STRUCTURE AND THE LOADING}

\subsection{Description of the structure}

An example of a single frame that is a part of a family house structure is considered (fig. 1). It is a two span three-storey frame and the two spans are of 6 and 7.20 $\mathrm{m}$ respectively. The storey height is $3 \mathrm{~m}$. The specific feature of the structure is that the top floor is shifted by $1.20 \mathrm{~m}$ because of architectural reasons.

Thus, the beam at the floor below supports the two columns at the façades. The soil conditions are not taken into account and the frame supports are considered as fixed. The dimensions of the beams are $25 / 60 \mathrm{~cm}$, and the columns are $25 / 40 \mathrm{~cm}$. The properties of concrete grade $\mathrm{C} 25 / 30$ are used in the calculations

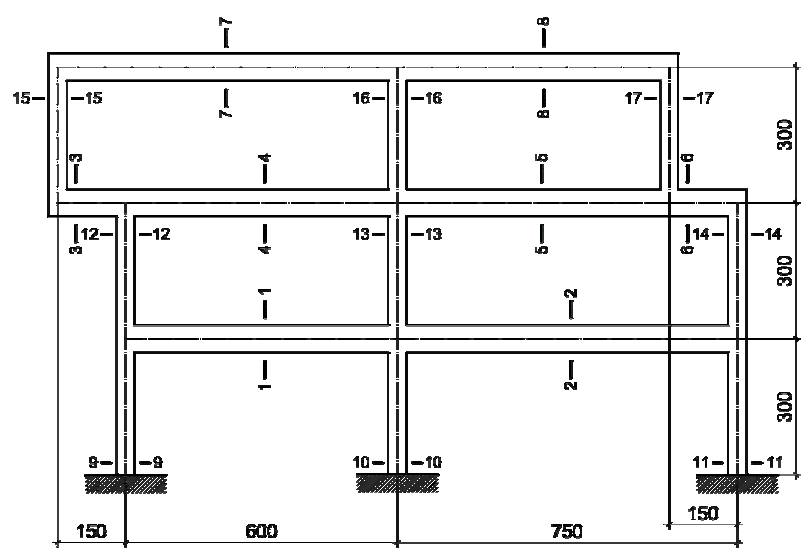

Figure 1: Frame 


\subsection{Description of the loading}

The loads are as follows: Self-weight - it is automatically calculated by the software; Wall on the beams - 14,62 kN/m; Load on the beams due to the corresponding part of the slab $-27,12 \mathrm{kN} / \mathrm{m}$; Live load on the beams due to the corresponding part of the slab $9,0 \mathrm{kN} / \mathrm{m}$.

Only static analysis of the frame is carried out. The coefficient is $\psi=1$ as the results are used only for the comparison of three different ways of loading.

The construction stages considered in the analysis correspond to the construction schedule presented below.

\begin{tabular}{|c|l|}
\hline day & \\
\hline 0 & $\begin{array}{l}\text { Disassembling of the formwork of the 1 floor } \\
\text { after the concrete has aged for 28 days }\end{array}$ \\
\hline 7 & $\begin{array}{l}\text { Constructed formwork and structure of the } \\
\text { second floor }\end{array}$ \\
\hline 35 & Disassembling of the second floor of the frame \\
\hline 42 & $\begin{array}{l}\text { Constructed formwork and structure of the third } \\
\text { floor }\end{array}$ \\
\hline 70 & Disassembling of the third floor of the frame \\
\hline 77 & The frame is loaded by the masonry walls \\
\hline 105 & The frame is loaded by live load and snow \\
\hline
\end{tabular}

\section{NUMERICAL MODEL}

The analysis software SAP2000 [1] is used for modelling and structural calculations. The beams and the columns are modelled with Frame elements with rectangular sections. Elements nodes are defined at 50 $\mathrm{cm}$ distance (fig.2).

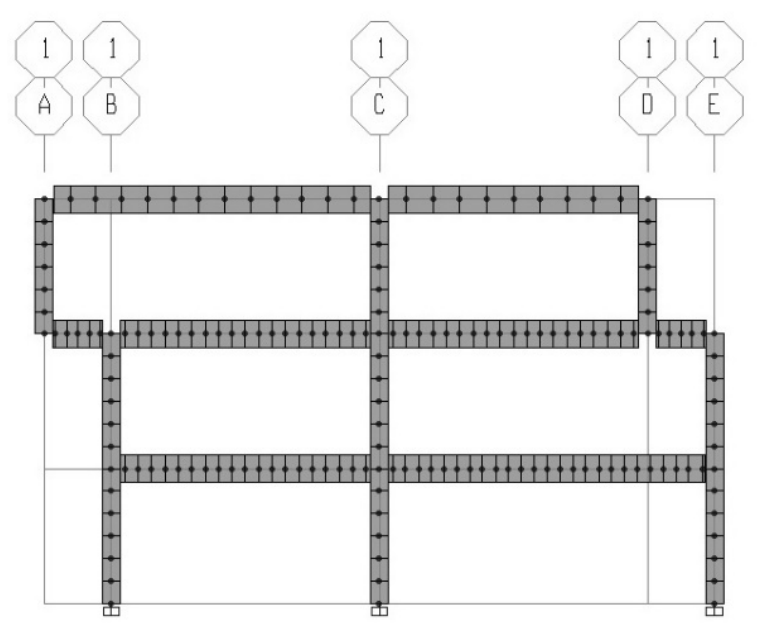

Figure 2: Model

Construction stages where the formwork transfers the loading from the already concreted structural part to the structure below are modelled by means of weightless frame elements. The modelling does not aim to investigate the influence of the formwork on the structural behaviour. The purpose of the modelling is to study the structural behaviour during the sequential construction stages and the influence of separate factors - creep, shrinkage, change of modulus of elasticity and combination of those factors, on the structural behaviour.

\subsection{Three different variants of defining the loading are considered and modelled numerically:}

3.1.1 The loads are defined and combined in the way that is usual in engineering - they act simultaneously on the structure that is idealized in the numerical (most often finite element) model. The main assumptions are as follows: deformations due to the self-weight of the structural elements during construction are not taken into account and it is also not considered that the rest of the loads are applied to the structure after the elements are deformed due to their self-weight. The results are presented in the following tables under the name COMBO.

3.1.2 The loads (walls and live load) are applied to the already constructed structure that is deformed because of the self-weight in the second variant. The results are presented in the following tables under the name STAGES.

3.1.3 The third variant is similar to the second one but the time dependent material properties (TDMP) of the concrete are taken into account. Those properties are introduced in the software according to the definitions and recommendations presented in CEB-FIP 90 [2]. The results are presented in the tables below under the name of STAGES+TDMP.

The load cases and their combinations are same for three variants of loading.

\subsection{Main relations for modelling the TDMP that are defined in CEB-FIP 90 [2] and used by SAP2000}

The main relations defined in CEB-FIP 90 that are used in the analysis carried out by means of SAP2000 software are presented in the text below.

3.2.1 Elastic deformation. That deformation is presented in the form $E_{c i}(t)=E_{c i} \sqrt{\beta_{c c}(t)}$, where $E_{c i}(t)$ is the modulus of elasticity for concrete at day $t$, and $E_{c i}$ is the modulus of elasticity at the 28th day, $\beta_{c c}(t)=\exp \left\{s .\left[1-(28 / t)^{0.5}\right]\right\}$ is factor depending on the age of concrete and cement type. The coefficient $s$ that corresponds to the concrete grade is introduced in the software. For concrete grade $\mathrm{C} 25 / 30$ with $f_{c m}=33 M P a \quad s=0.25$. The analysis and the conclusions are carried out assuming that the formwork for the previous construction stage is removed before constructing the next stage. 
3.2.2 Creep of concrete. For constant stress at time $t_{0}$ the deformation is $\varepsilon_{c c}=\frac{\sigma_{c}\left(t_{0}\right)}{E_{c i}} \phi\left(t, t_{0}\right)$, where $\sigma_{c}\left(t_{0}\right)$ is the stress that corresponds to the age of loading $t_{0}, \phi\left(t, t_{0}\right)$ is creep factor that can be calculated as $\phi\left(t, t_{0}\right)=\beta_{c}\left(t-t_{0}\right) \phi_{0}$, and $\beta_{c}$ is factor, describing the creep in the period after the loading, $\mathbf{t}$ [days] is the age of the concrete, $t_{0}$ is the age of the concrete at the time of loading:

$$
\begin{aligned}
& \phi_{0}=\phi_{R H} \beta\left(f_{c m}\right) \beta\left(t_{0}\right), \\
& \phi_{R H}=1+\frac{1-\left(\frac{R H}{R H_{0}}\right)}{0.46\left(\frac{h}{h_{0}}\right)^{\frac{1}{3}}}, \\
& \beta\left(f_{c m}\right)=\frac{5.3}{\sqrt{\frac{f_{c m}}{f_{c m 0}}}}, \\
& \beta\left(t_{0}\right)=\frac{1}{0.1+\left(\frac{t_{0}}{t_{1}}\right)^{0.2}} .
\end{aligned}
$$

All the quantities are defined in the text above. The relation between the creep and time is given by:

$$
\begin{aligned}
& \beta\left(t-t_{0}\right)=\left[\frac{\left(t-t_{0}\right) / t_{1}}{\beta_{H}+\left(t-t_{0}\right) / t_{1}}\right] \\
& \beta_{H}=150\left\{1+\left(1.2 \frac{R H}{R H_{0}}\right)^{18}\right\} \frac{h}{h_{0}}+250 \leq 1500
\end{aligned}
$$

Where $t_{1}=1$ day, $R H_{0}=1000$ and $h_{0}=100$. The relative humidity [\%] of the environment is introduced in the software as input data. The relative humidity is assumed in the analysis as equal to $80 \%$ that corresponds to open environment according to CEB- FIP 90.

\subsubsection{Shrinkage of concrete:}

$$
\varepsilon_{c s}\left(t, t_{s}\right)=\varepsilon_{c s o} \cdot \beta_{s}\left(t-t_{s}\right) \text {, where } \varepsilon_{c s o} \text { is }
$$
notional shrinkage coefficient, $\beta_{s}$ is factor, describing the shrinkage process related to time, $\mathbf{t}$ is the age of the concrete and $t_{s}$ is the age of the concrete when the shrinkage begins. Notional shrinkage coefficient can be calculated according to $\varepsilon_{c s o}=\varepsilon_{s}\left(f_{c m}\right) \beta_{R H}$,

$\varepsilon_{s}\left(f_{c m}\right)=\left[160+10 \beta_{s c}\left(9-\frac{f_{c m}}{f_{c m o}}\right)\right]$,

where $f_{c m}$ is the main concrete compression strength [MPa] at the age of 28 days, $f_{c m o}$ is assumed $10 \mathrm{MPa}, \beta_{s c}$ is factor of magnitude between 4 and 8 , that depends on the cement type. The last factor is assumed as equal to 5 in the analysis.

$$
\begin{aligned}
& \beta_{R H}=-1.55 \beta_{\text {sRH }} \rightarrow 40 \% \leq R H<99 \% \\
& \beta_{R H}=0.25 \rightarrow R H>99 \%
\end{aligned}
$$

where $\beta_{s R H}=1-\left(\frac{R H}{R H_{0}}\right)^{3}$, where $\mathbf{R H}$ is the relative humidity [\%] of the environment (atmosphere) and $R H_{0}$ is $100 \%$. The relation between shrinkage and time is given by: $\beta_{s}\left(t-t_{s}\right)=\sqrt{\frac{\left(t-t_{s}\right) / t_{1}}{350\left(h / h_{0}\right)+\left(t+t_{s}\right) / t_{1}}}$,

where $\mathbf{h}$ is the notational size of member $[\mathrm{mm}]$ that is given by $h=\frac{2 A_{c}}{u}$, where $A_{c}$ is the cross section area, and $\mathbf{u}$ is the parameter of the cross section part subjected to shrinkage; $h_{0}=100 \mathrm{~mm}$ and $t_{1}=1$ day.

\section{ANALYSIS AND COMPARISON OF THE RESULTS}

The main results of the calculations of three variants of modelling are compared. The loads are the same for all the models but the load cases are defined in different way. The results present the vertical and horizontal displacements and the internal forces at 17 particular sections of the frame elements. Those sections are shown on fig.1. The results are compared and the comparison is analyzed in order to show the influence of the construction stages and the time dependent material properties on the distribution of forces and displacements that correspond to particular load values:

\subsection{Elastic analysis (COMBO)}

The displacements and the internal forces in specific sections of the frame elements are shown below. 


\begin{tabular}{|c|c|c|c|c|c|c|c|c|c|c|c|c|c|c|c|c|c|}
\hline & 1 & 2 & 3 & 4 & 5 & 6 & 7 & 8 & 9 & 10 & 11 & 12 & 13 & 14 & 15 & 16 & 17 \\
\hline u3 [m] & \begin{tabular}{|l|}
$-0,002$ \\
\end{tabular} & $-0,005$ & $-0,003$ & $-0,001$ & $-0,008$ & \begin{tabular}{|c|}
$-0,005$ \\
\end{tabular} & $-0,008$ & $-0,005$ & 0,000 & 0,000 & 0,000 & $-0,001$ & \begin{tabular}{l|l|}
$-0,002$ \\
\end{tabular} & \begin{tabular}{|c|}
$-0,001$ \\
\end{tabular} & $-0,004$ & $-0,002$ & $-0,005$ \\
\hline u1 [m] & 0,000 & 0,000 & $-0,002$ & $-0,002$ & $-0,002$ & \begin{tabular}{|l|}
$-0,002$ \\
\end{tabular} & $-0,003$ & $-0,003$ & 0,000 & 0,000 & 0,000 & $-0,002$ & $-0,002$ & $-0,001$ & 004 & $-0,003$ & $-0,003$ \\
\hline 1 [kNm] & 86,64 & 148,59 & 25,58 & 5,01 & 192,01 & \begin{tabular}{|l|}
215,19 \\
\end{tabular} & 150,74 & 64,27 & $-26,20$ & $-12,06$ & 30,64 & $-3,34$ & 63,52 & $-95,32$ & 68,85 & 4,43 & $-8,80$ \\
\hline $\mathrm{Q}[\mathrm{kN}]$ & $-13,73$ & $-3,11$ & \begin{tabular}{|l|}
152,65 \\
\end{tabular} & $-50,09$ & $-23,49$ & \begin{tabular}{|l|}
191,73 \\
\end{tabular} & $-12,84$ & 10,73 & $-24,09$ & $-9,54$ & 33,63 & $-21,54$ & $-55,31$ & \begin{tabular}{|l|}
76,86 \\
\end{tabular} & $-17,31$ & $-23,80$ & 41,10 \\
\hline $\mathrm{N}[\mathrm{kN}]$ & $-2,54$ & 43,23 & 17,31 & $-4,24$ & $-35,75$ & \begin{tabular}{|l|}
$-76,85$ \\
\end{tabular} & $-17,31$ & $-41,10$ & $-574,75$ & $-1190,94$ & $-473,95$ & $-416,50$ & $-771,60$ & $-274,71$ & $-146,41$ & $-382,03$ & $-94,54$ \\
\hline
\end{tabular}

\subsection{The results corresponding to numerical modelling of the construction stages (STAGES)}

\begin{tabular}{|c|c|c|c|c|c|c|c|c|c|c|c|c|c|c|c|c|c|}
\hline & 1 & 2 & 3 & 4 & 5 & 6 & 7 & 8 & 9 & 10 & 11 & 12 & 13 & 14 & 15 & 16 & 17 \\
\hline u3 (105)[m] & $-0,002$ & $-0,006$ & $-0,003$ & $-0,001$ & $-0,008$ & $-0,005$ & $-0,008$ & $-0,004$ & 0,000 & 0,000 & 0,000 & $-0,001$ & $-0,002$ & $-0,001$ & $-0,003$ & $-0,002$ & $-0,003$ \\
\hline u1 (105)[m] & 0,000 & 0,000 & $-0,002$ & $-0,002$ & $-0,002$ & $-0,002$ & $-0,003$ & $-0,003$ & 0,000 & 0,000 & 0,000 & $-0,001$ & $-0,002$ & $-0,001$ & $-0,003$ & $-0,002$ & $-0,003$ \\
\hline $\mathrm{M}(105)[\mathrm{kNm}]$ & 90,29 & 157,99 & 32,67 & 6,10 & 210,52 & 211,11 & 152,70 & 69,40 & $-30,28$ & $-15,87$ & 36,48 & $-8,23$ & 65,62 & $-97,68$ & 65,72 & $-3,18$ & $-12,59$ \\
\hline Q (105) $[k N]$ & $-8,89$ & $-7,41$ & 151,66 & $-42,67$ & $-30,72$ & 185,68 & $-11,85$ & 11,94 & $-27,80$ & $-12,80$ & 40,60 & $-10,65$ & $-51,42$ & 62,07 & $-13,22$ & $-14,31$ & 27,53 \\
\hline $\mathrm{N}(105)[\mathrm{kN}]$ & $-17,15$ & 21,47 & 13,22 & 2,57 & $-34,54$ & $-62,07$ & $-13,22$ & $-27,53$ & $-561,49$ & $-1214,54$ & $-463,62$ & $-408,09$ & $-786,05$ & $-268,67$ & $-145,42$ & $-381,84$ & $-95,73$ \\
\hline
\end{tabular}

Comparison of the differences in \% between the results of two solutions is presented in the table below.

\section{COMBO/STAGES}

\begin{tabular}{|l|r|r|r|r|r|r|r|r|r|r|r|r|r|r|r|r|r|}
\hline & 1 & 2 & 3 & 4 & 5 & 6 & 7 & 8 & 9 & 10 & 11 & 12 & 13 & 14 & 15 & 16 & 17 \\
\hline $\mathrm{u} 3(105)[\mathrm{m}]$ & $3 \%$ & $8 \%$ & $5 \%$ & $18 \%$ & $8 \%$ & $7 \%$ & $3 \%$ & $27 \%$ & & & & $6 \%$ & $8 \%$ & $14 \%$ & $4 \%$ & $27 \%$ & $38 \%$ \\
\hline $\mathrm{u} 1(105)[\mathrm{m}]$ & $20 \%$ & $38 \%$ & $13 \%$ & $14 \%$ & $14 \%$ & $14 \%$ & $22 \%$ & $22 \%$ & & & & $15 \%$ & $9 \%$ & $40 \%$ & $18 \%$ & $25 \%$ & $27 \%$ \\
\hline $\mathrm{M} \mathrm{(105)}[\mathrm{kNm}]$ & $4 \%$ & $6 \%$ & $28 \%$ & $22 \%$ & $10 \%$ & $2 \%$ & $1 \%$ & $8 \%$ & $16 \%$ & $32 \%$ & $19 \%$ & $146 \%$ & $3 \%$ & $2 \%$ & $5 \%$ & $28 \%$ & $43 \%$ \\
\hline $\mathrm{Q}(105)[\mathrm{kN}]$ & $35 \%$ & $138 \%$ & $1 \%$ & $15 \%$ & $31 \%$ & $3 \%$ & $8 \%$ & $11 \%$ & $15 \%$ & $34 \%$ & $21 \%$ & $51 \%$ & $7 \%$ & $19 \%$ & $24 \%$ & $40 \%$ & $33 \%$ \\
\hline $\mathrm{N}(105)[\mathrm{kN}]$ & $575 \%$ & $50 \%$ & $24 \%$ & $39 \%$ & $3 \%$ & $19 \%$ & $24 \%$ & $33 \%$ & $2 \%$ & $2 \%$ & $2 \%$ & $2 \%$ & $2 \%$ & $2 \%$ & $1 \%$ & $0 \%$ & $1 \%$ \\
\hline
\end{tabular}

The two sets of results reveal differences in the elastic displacements and the internal forces. It can be seen that the bending moments in the beams are bigger when the construction stages are taken into account, while the shear and axial forces in the vertical elements at the top storey decrease.
The variation of the considered quantities - vertical and horizontal displacements, bending moments, axial and shear forces corresponding to the construction stages, is presented in the following tables.

Vertikal displacements (elastic)

\begin{tabular}{|c|r|r|r|r|r|r|r|r|r|r|r|r|r|r|r|r|r|}
\hline day & 1 & 2 & 3 & 4 & 5 & 6 & 7 & 8 & 9 & 10 & 11 & 12 & 13 & 14 & 15 & 16 & 17 \\
\hline 0 & $-0,001$ & $-0,003$ & & & & & & & 0,000 & 0,000 & 0,000 & & & & & & \\
\hline 7 & $-0,002$ & $-0,007$ & & & & & & & 0,000 & 0,000 & 0,000 & & & & & & \\
\hline 35 & $-0,001$ & $-0,003$ & 0,000 & $-0,001$ & $-0,004$ & $-0,002$ & & & 0,000 & 0,000 & 0,000 & 0,000 & 0,000 & 0,000 & & & \\
\hline 42 & $-0,001$ & $-0,003$ & 0,000 & $-0,002$ & $-0,007$ & $-0,004$ & & & 0,000 & 0,000 & 0,000 & 0,000 & $-0,001$ & 0,000 & & & \\
\hline 70 & $-0,001$ & $-0,003$ & $-0,002$ & 0,000 & $-0,005$ & $-0,003$ & $-0,005$ & $-0,002$ & 0,000 & 0,000 & 0,000 & 0,000 & $-0,001$ & 0,000 & $-0,002$ & $-0,002$ & $-0,001$ \\
\hline 77 & $-0,002$ & $-0,005$ & $-0,002$ & $-0,001$ & $-0,007$ & $-0,004$ & $-0,005$ & $-0,002$ & 0,000 & 0,000 & 0,000 & $-0,001$ & $-0,001$ & 0,000 & $-0,002$ & $-0,001$ & $-0,002$ \\
\hline 105 & $-0,002$ & $-0,006$ & $-0,003$ & $-0,001$ & $-0,008$ & $-0,005$ & $-0,008$ & $-0,004$ & 0,000 & 0,000 & 0,000 & $-0,001$ & $-0,002$ & $-0,001$ & $-0,003$ & $-0,002$ & $-0,003$ \\
\hline
\end{tabular}

Horizontal displacements (elastic)

\begin{tabular}{|c|r|r|r|r|r|r|r|r|r|r|r|r|r|r|r|r|r|r|}
\hline day & 1 & 2 & 3 & 4 & 5 & 6 & 7 & 8 & 9 & 10 & 11 & 12 & 13 & 14 & 15 & 16 & 17 \\
\hline 0 & 0,000 & 0,000 & & & & & & & 0,000 & 0,000 & 0,000 & & & & & & \\
\hline 7 & 0,000 & $-0,001$ & & & & & & & 0,000 & 0,000 & 0,000 & & & & & & \\
\hline 35 & 0,000 & 0,000 & 0,000 & 0,000 & 0,000 & 0,000 & & & 0,000 & 0,000 & 0,000 & 0,000 & 0,000 & 0,000 & & & \\
\hline 42 & 0,000 & 0,000 & $-0,001$ & $-0,001$ & $-0,001$ & $-0,001$ & & & 0,000 & 0,000 & 0,000 & $-0,001$ & $-0,001$ & 0,000 & & & \\
\hline 70 & 0,000 & 0,000 & $-0,001$ & $-0,001$ & $-0,001$ & $-0,001$ & $-0,001$ & $-0,001$ & 0,000 & 0,000 & 0,000 & $-0,001$ & $-0,001$ & 0,000 & $-0,002$ & $-0,002$ & $-0,001$ \\
\hline 77 & 0,000 & 0,000 & $-0,001$ & $-0,001$ & $-0,001$ & $-0,001$ & $-0,002$ & $-0,002$ & 0,000 & 0,000 & 0,000 & $-0,001$ & $-0,001$ & 0,000 & $-0,002$ & $-0,001$ & $-0,002$ \\
\hline 105 & 0,000 & 0,000 & $-0,002$ & $-0,002$ & $-0,002$ & $-0,002$ & $-0,003$ & $-0,003$ & 0,000 & 0,000 & 0,000 & $-0,001$ & $-0,002$ & $-0,001$ & $-0,003$ & $-0,002$ & $-0,003$ \\
\hline
\end{tabular}

Bending moments

Bending moments
\begin{tabular}{|c|r|r|r|r|r|r|r|r|r|r|r|r|r|r|r|r|r|r|r|}
\hline day & 1 & 2 & 3 & 4 & 5 & 6 & 7 & 8 & 9 & 10 & 11 & 12 & 13 & 14 & 15 & 16 & 17 \\
\hline 0 & 51,52 & 99,53 & & & & & & & $-17,89$ & $-12,41$ & 25,65 & & & & & & \\
\hline 7 & 103,33 & 199,46 & & & & & & & $-35,80$ & $-28,43$ & 51,32 & & & & & & \\
\hline 35 & 50,23 & 95,01 & 0,00 & 40,95 & 103,33 & 59,23 & & & $-17,30$ & $-12,24$ & 23,53 & 14,43 & 19,97 & $-43,64$ & & & \\
\hline 42 & 48,95 & 90,50 & 0,00 & 82,20 & 207,09 & 119,41 & & & $-16,70$ & $-10,26$ & 21,41 & 28,84 & 39,57 & $-87,26$ & & & \\
\hline 70 & 53,27 & 93,26 & 26,79 & $-3,98$ & 132,68 & 130,82 & 104,02 & 49,43 & $-19,27$ & $-10,30$ & 23,12 & $-11,33$ & 42,03 & $-59,12$ & 42,28 & $-5,81$ & $-9,87$ \\
\hline 77 & 75,33 & 133,80 & 23,30 & 14,93 & 172,90 & 160,93 & 102,94 & 46,94 & $-25,52$ & $-14,29$ & 31,49 & $-2,14$ & 50,43 & $-78,78$ & 43,97 & $-2,20$ & $-8,28$ \\
\hline 105 & 90,29 & 157,99 & 32,67 & 6,10 & 210,52 & 211,11 & 152,70 & 69,40 & $-30,28$ & $-15,87$ & 36,48 & $-8,23$ & 65,62 & $-97,68$ & 65,72 & $-3,18$ & $-12,59$ \\
\hline
\end{tabular}




\begin{tabular}{|c|c|c|c|c|c|c|c|c|c|c|c|c|c|c|c|c|c|}
\hline \multicolumn{18}{|c|}{ hear forces } \\
\hline day & 1 & 2 & 3 & 4 & 5 & 6 & 7 & 8 & 9 & 10 & 11 & 12 & 13 & 14 & 15 & \begin{tabular}{l|}
16 \\
\end{tabular} & 17 \\
\hline 0 & 2,49 & $-7,05$ & & & & & & & $-16,43$ & $-12,46$ & 28,89 & & & & & & \\
\hline 7 & 9,50 & $-9,36$ & & & & & & & $-32,85$ & $-24,92$ & 57,77 & & & & & & \\
\hline 35 & $-1,27$ & $-5,35$ & 0,00 & 2,69 & \begin{tabular}{|c|}
$-11,41$ \\
\end{tabular} & 53,41 & & & \begin{tabular}{|l|}
$-15,17$ \\
\end{tabular} & $-10,50$ & 26,25 & \begin{tabular}{|c|}
$-10,49$ \\
\end{tabular} & \begin{tabular}{|l|}
$-13,46$ \\
\end{tabular} & 23,94 & & & \\
\hline 42 & $-5,05$ & $-3,65$ & 3,88 & 10,17 & $-18,12$ & 114,75 & & & $-15,07$ & $-8,53$ & 23,60 & $-20,97$ & $-26,91$ & 47,88 & & & \\
\hline 70 & $-3,28$ & $-6,22$ & 103,81 & $-24,67$ & $-22,79$ & 113,41 & $-7,27$ & 8,31 & $-17,70$ & $-8,27$ & 25,97 & 0,67 & $-30,46$ & 29,79 & $-6,20$ & $-5,10$ & 11,30 \\
\hline 77 & $-6,19$ & $-6,72$ & 104,38 & $-29,57$ & $-24,71$ & 141,75 & $-7,84$ & 7,87 & $-23,39$ & $-11,70$ & 35,09 & $-9,50$ & $-38,70$ & 48,19 & $-8,28$ & $-9,63$ & 17,89 \\
\hline 105 & $-8,89$ & $-7,41$ & 151,66 & $-42,67$ & \begin{tabular}{|l|}
$-30,72$ \\
\end{tabular} & 185,68 & $-11,85$ & 11,94 & $-27,80$ & $-12,80$ & 40,60 & \begin{tabular}{|c|}
$-10,65$ \\
\end{tabular} & \begin{tabular}{|c|}
$-51,42$ \\
\end{tabular} & 62,07 & \begin{tabular}{|l|}
$-13,22$ \\
\end{tabular} & \begin{tabular}{|c|}
$-14,31$ \\
\end{tabular} & 27,53 \\
\hline
\end{tabular}

\begin{tabular}{|c|c|c|c|c|c|c|c|c|c|c|c|c|c|c|c|c|c|}
\hline \multicolumn{18}{|c|}{ Axial forces } \\
\hline day & 1 & 2 & 3 & 4 & 5 & 6 & 7 & 8 & 9 & 10 & 11 & 12 & 13 & 14 & 15 & 16 & 17 \\
\hline 0 & $-16,43$ & $-28,89$ & & & & & & & $-79,09$ & $-252,83$ & $-107,30$ & & & & & & \\
\hline 7 & $-31,33$ & $-55,38$ & & & & & & & $-151,14$ & $-498,93$ & $-207,65$ & & & & & & \\
\hline 35 & $-5,27$ & $-2,31$ & 0,00 & $-10,49$ & $-23,94$ & $-23,94$ & & & $-213,44$ & $-495,10$ & $-216,22$ & $-124,32$ & $-241,49$ & $-100,97$ & & & \\
\hline 42 & 5,90 & 24,28 & 0,00 & $-19,96$ & $-45,64$ & $-45,72$ & & & $-340,94$ & $-730,65$ & $-318,16$ & $-248,05$ & $-482,52$ & $-201,21$ & & & \\
\hline 70 & $-18,36$ & 3,82 & 6,20 & 6,86 & \begin{tabular}{|l|}
$-18,49$ \\
\end{tabular} & $-29,79$ & $-6,20$ & $-11,30$ & $-341,23$ & $-747,41$ & $-275,34$ & $-250,12$ & $-494,93$ & $-160,96$ & $-97,57$ & $-257,79$ & $-65,12$ \\
\hline 77 & $-13,90$ & 13,10 & 8,27 & $-1,22$ & $-30,30$ & $-48,19$ & $-8,23$ & $-17,89$ & $-441,72$ & $-963,21$ & $-375,32$ & \begin{tabular}{|l|}
$-312,61$ \\
\end{tabular} & \begin{tabular}{|l|}
$-601,46$ \\
\end{tabular} & \begin{tabular}{|l|}
$-211,23$ \\
\end{tabular} & $-98,14$ & \begin{tabular}{|l|}
$-257,66$ \\
\end{tabular} & $-64,68$ \\
\hline 105 & $-17,15$ & 21,47 & \begin{tabular}{l|}
13,22 \\
\end{tabular} & 2,57 & $-34,54$ & \begin{tabular}{|l|}
$-62,07$ \\
\end{tabular} & $-13,22$ & $-27,53$ & $-561,49$ & $-1214,54$ & $-463,62$ & $-408,09$ & $-786,05$ & \begin{tabular}{|l|}
$-268,67$ \\
\end{tabular} & $-145,42$ & $-381,84$ & $-95,73$ \\
\hline
\end{tabular}

\subsection{Model that includes both the construction stages and TDMP of concrete.}

\begin{tabular}{|c|c|c|c|c|c|c|c|c|c|c|c|c|c|c|c|c|c|}
\hline & 1 & 2 & 3 & 4 & 5 & 6 & 7 & 8 & 9 & 10 & 11 & 12 & 13 & 14 & 15 & 16 & 17 \\
\hline u3 (105) [m] & $-0,004$ & $-0,011$ & $-0,005$ & $-0,001$ & $-0,014$ & $-0,009$ & $-0,011$ & $-0,005$ & 0,000 & 0,000 & 0,000 & $-0,001$ & $-0,003$ & $-0,001$ & $-0,005$ & $-0,002$ & $-0,004$ \\
\hline u1 (105)[m] & 0,000 & $-0,001$ & $-0,002$ & $-0,002$ & $-0,003$ & $-0,003$ & $-0,003$ & $-0,004$ & 0,000 & 0,000 & 0,000 & $-0,002$ & $-0,003$ & $-0,001$ & $-0,004$ & $-0,003$ & $-0,003$ \\
\hline $\mathrm{M}(105)[\mathrm{kNm}]$ & 91,52 & 157,90 & 30,25 & 3,01 & 214,06 & 182,97 & 151,94 & 69,62 & $-24,74$ & $-15,84$ & 30,93 & $-10,38$ & 67,32 & $-96,46$ & 63,49 & $-5,99$ & $-13,92$ \\
\hline $\mathrm{Q}(105)[\mathrm{kN}]$ & $-6,03$ & $-9,61$ & 150,64 & $-40,38$ & $-33,25$ & 208,89 & $-10,83$ & 11,70 & $-24,41$ & $-12,71$ & 37,11 & $-8,41$ & $-52,16$ & 60,56 & $-13,23$ & $-10,36$ & 23,65 \\
\hline $\mathrm{N}(105)[\mathrm{kN}]$ & $-16,01$ & 23,45 & 13,29 & 4,89 & $-36,91$ & $-60,56$ & $-13,29$ & $-23,65$ & $-555,22$ & $-1225,64$ & $-458,68$ & $-404,88$ & $-792,11$ & $-265,96$ & $-144,39$ & $-383,06$ & $-95,51$ \\
\hline
\end{tabular}

The differences in the results are presented in the table below:

\section{COMBO/STAGES+TDMP}

\begin{tabular}{|c|c|c|c|c|c|c|c|c|c|c|c|c|c|c|c|c|c|}
\hline & 1 & 2 & 3 & 4 & 5 & 6 & 7 & 8 & \begin{tabular}{l|}
9 \\
\end{tabular} & \begin{tabular}{l|l}
10 \\
\end{tabular} & \begin{tabular}{l|l}
11 \\
\end{tabular} & 12 & \begin{tabular}{l|}
13 \\
\end{tabular} & 14 & 15 & 16 & 17 \\
\hline u3 (105)[m] & $84 \%$ & $105 \%$ & $37 \%$ & $89 \%$ & $90 \%$ & $87 \%$ & $44 \%$ & $3 \%$ & & & & $72 \%$ & $55 \%$ & $69 \%$ & $40 \%$ & $5 \%$ & $23 \%$ \\
\hline u1 (105)[m] & $53 \%$ & $250 \%$ & $8 \%$ & $3 \%$ & $28 \%$ & $37 \%$ & $1 \%$ & $6 \%$ & & & & $9 \%$ & $37 \%$ & $15 \%$ & $8 \%$ & $2 \%$ & $1 \%$ \\
\hline $\mathrm{M}(105)[\mathrm{kNm}]$ & $6 \%$ & $6 \%$ & $18 \%$ & $40 \%$ & $11 \%$ & $15 \%$ & $1 \%$ & $8 \%$ & $6 \%$ & $31 \%$ & $1 \%$ & $211 \%$ & $6 \%$ & $1 \%$ & $8 \%$ & $35 \%$ & $58 \%$ \\
\hline $\mathrm{Q}(105)[\mathrm{kN}]$ & $56 \%$ & $209 \%$ & $1 \%$ & $19 \%$ & $42 \%$ & $9 \%$ & $16 \%$ & $9 \%$ & $1 \%$ & $33 \%$ & $10 \%$ & $61 \%$ & $6 \%$ & $21 \%$ & $24 \%$ & $56 \%$ & $42 \%$ \\
\hline $\mathrm{N}(105)[\mathrm{kN}]$ & $30 \%$ & $46 \%$ & $23 \%$ & $15 \%$ & $3 \%$ & $21 \%$ & $23 \%$ & $42 \%$ & $3 \%$ & $3 \%$ & $3 \%$ & $3 \%$ & $3 \%$ & $3 \%$ & $1 \%$ & $0 \%$ & $1 \%$ \\
\hline
\end{tabular}

\section{STAGES/STAGES+TDMP}

\begin{tabular}{|c|c|c|c|c|c|c|c|c|c|c|c|c|c|c|c|c|c|}
\hline & 1 & 2 & 3 & 4 & 5 & 6 & 7 & 8 & 9 & 10 & 11 & 12 & 13 & 14 & 15 & 16 & 17 \\
\hline $3(105)$ & $5 \%$ & $89 \%$ & $5 \%$ & $29 \%$ & $6 \%$ & $75 \%$ & $8 \%$ & $3 \%$ & & & & $33 \%$ & $8 \%$ & $6 \%$ & $6 \%$ & $44 \%$ & $4 \%$ \\
\hline u1 (105)[m] & $28 \%$ & $53 \%$ & $25 \%$ & $20 \%$ & $49 \%$ & $59 \%$ & $28 \%$ & $36 \%$ & & & & $28 \%$ & $50 \%$ & $41 \%$ & $31 \%$ & $31 \%$ & $35 \%$ \\
\hline $\mathrm{M}(105)[\mathrm{kNn}$ & $1 \%$ & $0 \%$ & $7 \%$ & $51 \%$ & $2 \%$ & $13 \%$ & $0 \%$ & $0 \%$ & & $\%$ & 70 & $26 \%$ & $3 \%$ & $1 \%$ & $3 \%$ & $88 \%$ & $11 \%$ \\
\hline $\mathrm{Q}(105)[\mathrm{kN}]$ & $32 \%$ & $30 \%$ & $1 \%$ & $5 \%$ & $8 \%$ & $13 \%$ & $9 \%$ & $2 \%$ & $12 \%$ & $1 \%$ & $9 \%$ & $21 \%$ & $1 \%$ & $2 \%$ & $0 \%$ & $28 \%$ & $14 \%$ \\
\hline $\mathrm{N}(105)[\mathrm{kN}]$ & $7 \%$ & $9 \%$ & $1 \%$ & $90 \%$ & $7 \%$ & $2 \%$ & $1 \%$ & $14 \%$ & $1 \%$ & $1 \%$ & $1 \%$ & $1 \%$ & $1 \%$ & $1 \%$ & $1 \%$ & $0 \%$ & $0 \%$ \\
\hline
\end{tabular}

The comparison of the results of three variants of modelling and calculation of the structure show differences in the magnitudes of displacements and the internal forces. Taking into account the time dependent material properties in general results increase in the magnitude of all quantities. That increase is different at different structural elements.

Changes in vertical and horizontal displacements, bending moments, shear and axial forces when accounting for the construction stages and the TDMP of concrete are presented in the following tables.

Vertikal displacements (elastic)

\begin{tabular}{|c|c|c|c|c|c|c|c|c|c|c|c|c|c|c|c|c|c|}
\hline day & 1 & 2 & 3 & 4 & 5 & 6 & 7 & 8 & 9 & 10 & 11 & 12 & 13 & 14 & 15 & 16 & 17 \\
\hline 0 & $-0,001$ & $-0,003$ & & & & & & & 0,000 & 0,000 & 0,000 & & & & & & \\
\hline 7 & $-0,002$ & $-0,008$ & & & & & & & 0,000 & 0,000 & 0,000 & & & & & & \\
\hline 35 & $-0,003$ & $-0,008$ & 0,000 & $-0,001$ & $-0,004$ & $-0,002$ & & & 0,000 & 0,000 & 0,000 & 0,000 & 0,000 & 0,000 & & & \\
\hline 42 & $-0,003$ & $-0,007$ & 0,000 & $-0,002$ & $-0,009$ & $-0,005$ & & & 0,000 & 0,000 & 0,000 & $-0,001$ & $-0,001$ & 0,000 & & & \\
\hline 70 & $-0,003$ & $-0,007$ & $-0,002$ & $-0,001$ & $-0,011$ & $-0,007$ & $-0,005$ & \begin{tabular}{|l|}
$-0,002$ \\
\end{tabular} & 0,000 & 0,000 & 0,000 & $-0,001$ & $-0,002$ & $-0,001$ & $-0,002$ & $-0,001$ & $-0,001$ \\
\hline 77 & $-0,004$ & $-0,009$ & $-0,003$ & $-0,001$ & $-0,012$ & $-0,007$ & $-0,007$ & \begin{tabular}{|l|}
$-0,003$ \\
\end{tabular} & 0,000 & 0,000 & 0,000 & $-0,001$ & $-0,002$ & $-0,001$ & $-0,003$ & $-0,001$ & $-0,002$ \\
\hline 105 & $-0,004$ & $-0,011$ & $-0,005$ & $-0,001$ & $-0,014$ & $-0,009$ & $-0,011$ & $\mid-0,005$ & 0,000 & 0,000 & 0,000 & $-0,001$ & $-0,003$ & $-0,001$ & $-0,005$ & $-0,002$ & $-0,004$ \\
\hline
\end{tabular}




\begin{tabular}{|c|c|c|c|c|c|c|c|c|c|c|c|c|c|c|c|c|c|}
\hline day & 1 & & & 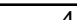 & & & & & & & & & & & & & \\
\hline & & & & & & & & & & & & & & & & & \\
\hline 0 & 0,000 & 0,000 & & & & & & & 0,000 & 0,000 & 0,000 & & & & & & \\
\hline 7 & $-0,001$ & $-0,001$ & & & & & & & 0,000 & 0,000 & 0,000 & & & & & & \\
\hline 35 & 0,000 & $-0,001$ & 0,000 & 0,000 & 0,000 & 0,000 & & & 0,000 & 0,000 & 0,000 & 0,000 & 0,000 & 0,000 & & & \\
\hline 42 & 0,000 & $-0,001$ & $-0,001$ & $-0,001$ & $-0,001$ & $-0,001$ & & & 0,000 & 0,000 & 0,000 & $-0,001$ & $-0,001$ & 0,001 & & & \\
\hline 70 & 0,000 & $-0,001$ & $-0,001$ & $-0,001$ & $-0,001$ & $-0,002$ & $-0,001$ & $-0,001$ & 0,000 & 0,000 & 0,000 & $-0,001$ & $-0,002$ & 0,000 & $-0,002$ & $-0,001$ & $-0,001$ \\
\hline 77 & 0,000 & $-0,001$ & $-0,002$ & $-0,002$ & $-0,002$ & $-0,002$ & $-0,002$ & $-0,002$ & 0,000 & 0,000 & 0,000 & $-0,001$ & $-0,002$ & 0,000 & $-0,003$ & $-0,002$ & $-0,002$ \\
\hline 105 & 0,000 & $-0,001$ & $-0,002$ & $-0,002$ & $-0,003$ & $-0,003$ & $-0,003$ & $-0,004$ & 0,000 & 0,000 & 0,000 & $-0,002$ & $-0,003$ & $-0,001$ & $-0,004$ & $-0,003$ & $-0,003$ \\
\hline
\end{tabular}

\begin{tabular}{|c|c|c|c|c|c|c|c|c|c|c|c|c|c|c|c|c|c|}
\hline day & 1 & 2 & 3 & 4 & 5 & 6 & 7 & 8 & 9 & 10 & 11 & 12 & 13 & 14 & 15 & 16 & 17 \\
\hline 0 & 51,52 & 99,53 & & & & & & & $-17,89$ & $-14,21$ & 25,65 & & & & & & \\
\hline 7 & 102,83 & 198,76 & & & & & & & $-35,00$ & $-28,63$ & 50,73 & & & & & & \\
\hline 35 & 49,10 & 93,13 & 0,00 & 40,94 & 103,29 & 59,19 & & & $-14,17$ & $-12,68$ & 20,83 & 14,43 & 19,80 & $-43,64$ & & & \\
\hline 42 & 49,75 & 92,85 & 0,00 & 82,13 & 207,81 & 122,76 & & & $-14,47$ & $-11,81$ & 23,53 & 26,55 & 38,36 & $-85,11$ & & & \\
\hline 70 & 54,40 & 94,57 & 25,43 & $-6,06$ & 131,89 & 115,59 & 103,91 & 49,38 & $-15,16$ & $-10,23$ & 24,02 & $-14,30$ & 41,42 & $-55,70$ & 42,35 & $-6,12$ & $-10,23$ \\
\hline 77 & 76,90 & 134,65 & 22,14 & 12,35 & 178,09 & 158,36 & 102,53 & 47,86 & $-21,18$ & $-14,55$ & 27,24 & $-4,60$ & 52,22 & $-78,05$ & 41,91 & $-5,47$ & $-9,81$ \\
\hline 105 & 91,52 & 157,90 & 30,25 & 3,01 & 214,06 & 182,97 & 151,94 & 69,62 & $-24,74$ & $-15,84$ & 30,93 & $-10,38$ & 67,32 & $-96,46$ & 63,49 & $-5,99$ & $-13,92$ \\
\hline
\end{tabular}

\begin{tabular}{|c|c|c|c|c|c|c|c|c|c|c|c|c|c|c|c|c|c|}
\hline day & 1 & 2 & 3 & 4 & 5 & 6 & 7 & 8 & 9 & 10 & 11 & 12 & 13 & 14 & 15 & 16 & 17 \\
\hline 0 & 2,49 & $-7,05$ & & & & & & & $-16,43$ & $-12,48$ & 28,89 & & & & & & \\
\hline 7 & 0,90 & $-9,92$ & & & & & & & $-32,19$ & $-24,97$ & 57,16 & & & & & & \\
\hline 35 & 0,85 & $-7,04$ & 0,00 & $-2,68$ & $-11,42$ & 53,41 & & & $-13,36$ & $-10,58$ & 23,95 & $-10,51$ & \begin{tabular}{|l|}
$-13,51$ \\
\end{tabular} & 24,06 & & & \\
\hline 42 & $-1,70$ & $-6,41$ & 3,91 & 0,92 & $-19,37$ & 98,16 & & & $-13,80$ & $-9,71$ & 19,99 & $-17,13$ & $-24,64$ & 41,77 & & & \\
\hline 70 & \begin{tabular}{|l|}
$-0,49$ \\
\end{tabular} & $-8,44$ & 103,92 & $-22,46$ & $-24,65$ & 40,18 & $-7,34$ & 8,50 & $-15,27$ & $-8,75$ & 19,31 & 3,71 & $-29,34$ & 25,65 & $-6,72$ & $-4,66$ & 11,38 \\
\hline 77 & \begin{tabular}{|l|}
$-3,45$ \\
\end{tabular} & $\begin{array}{l}-8,88 \\
\end{array}$ & 103,45 & $-26,78$ & $-27,86$ & 138,77 & $-6,91$ & 7,85 & $-20,84$ & \begin{tabular}{|c|}
$-11,59$ \\
\end{tabular} & 32,70 & $-6,63$ & $-39,12$ & 45,80 & $-7,91$ & $-5,01$ & 12,92 \\
\hline 105 & $-6,03$ & $-9,61$ & 150,64 & $-40,38$ & $-33,25$ & 208,89 & \begin{tabular}{|c|}
$-10,83$ \\
\end{tabular} & 11,70 & $-24,41$ & \begin{tabular}{|c|}
$-12,71$ \\
\end{tabular} & 37,11 & $-8,41$ & $-52,16$ & 60,56 & $-13,23$ & $-10,36$ & 23,65 \\
\hline
\end{tabular}

\begin{tabular}{|c|c|c|c|c|c|c|c|c|c|c|c|c|c|c|c|c|c|}
\hline \\
\hline day & 1 & 2 & 3 & 4 & 5 & 6 & 7 & 8 & 9 & 10 & 11 & 12 & 13 & 14 & 15 & 16 & 17 \\
\hline 0 & $-16,43$ & $-28,89$ & & & & & & & $-79,09$ & $-252,83$ & \begin{tabular}{|l|}
$-107,31$ \\
\end{tabular} & & & & & & \\
\hline 7 & $-30,68$ & $-54,79$ & & & & & & & $-150,43$ & $-500,20$ & \begin{tabular}{|l|}
$-207,09$ \\
\end{tabular} & & & & & & \\
\hline 35 & $-2,86$ & 0,06 & 0,00 & $-10,51$ & \begin{tabular}{|l|}
$-24,02$ \\
\end{tabular} & \begin{tabular}{|l|}
$-24,02$ \\
\end{tabular} & & & $-211,31$ & $-498,92$ & \begin{tabular}{|l|}
$-214,52$ \\
\end{tabular} & \begin{tabular}{|l|}
$-124,32$ \\
\end{tabular} & $-241,50$ & $-100,96$ & & & \\
\hline 42 & 3,33 & 18,25 & 0,00 & $-16,14$ & $-39,64$ & $-39,64$ & & & $-335,80$ & $-739,80$ & $-314,15$ & \begin{tabular}{|l|}
$-246,27$ \\
\end{tabular} & $-485,56$ & $-199,96$ & & & \\
\hline 70 & $-18,97$ & 1,63 & 6,72 & 10,42 & $\mid-14,27$ & $-25,65$ & $-6,72$ & $\mid-11,38$ & $-336,31$ & $\begin{array}{l}-756,22 \\
\end{array}$ & \begin{tabular}{|l|}
$-271,45$ \\
\end{tabular} & \begin{tabular}{|l|}
$-247,98$ \\
\end{tabular} & $-498,74$ & $-159,29$ & $-97,64$ & \begin{tabular}{|l|}
$-253,57$ \\
\end{tabular} & $-65,31$ \\
\hline 77 & \begin{tabular}{|l|}
$-14,22$ \\
\end{tabular} & 13,04 & 7,91 & 1,28 & \begin{tabular}{|l|}
$-32,82$ \\
\end{tabular} & $-45,74$ & $-7,91$ & \begin{tabular}{|l|}
$-12,92$ \\
\end{tabular} & $-435,26$ & $-974,81$ & \begin{tabular}{|l|}
$-370,57$ \\
\end{tabular} & $-308,89$ & $-608,18$ & $-208,24$ & $-97,21$ & $-258,51$ & $-64,67$ \\
\hline 105 & $-16,01$ & 23,45 & 13,29 & 4,89 & $-36,91$ & $-60,56$ & $-13,29$ & $\mid-23,65$ & $-555,22$ & $-1225,64$ & $-458,68$ & $-404,88$ & $-792,11$ & $-265,96$ & \begin{tabular}{|l|}
$-144,39$ \\
\end{tabular} & $-383,06$ & $-95,51$ \\
\hline
\end{tabular}

The graphic presentation of the results is not included in the present article due to its relatively large volume.

\section{CONCLUSIONS}

Taking into account the construction stages, they affect significantly the displacements and the internal forces of the structural elements. That influence is different on the different elements as well as on the separate internal forces in particular section. The differences depend also on the material type, the structural geometry, the type and sequence of loads' application and the climate, except the type and position of the particular element in consideration. The presented results of different ways of modelling and calculation of the structure show that the construction stages have a slight influence on the elements like end columns and short beams $(L<10$. hbeem), no matter if the TDMP of concrete are taken into account or not. However for longer beams $(\mathrm{L}>10 . \mathrm{hbeem})$, or the column at the middle of the frame the results show significant differences and the obtained displacements and internal forces are of bigger magnitude. There is the other group of elements as the transfer columns at the top storey where accounting for the construction stages results in decrease of the internal forces. The study of the example structure in the article show that construction stages including the time-dependent material properties shall be taken into account in the design. There are differences in the displacements and the internal forces in main structural elements that cannot be neglected.

It should be kept in mind that during the construction the structural response could result in displacements and internal forces of magnitudes that are higher than the ones the elements are designed for.

\section{LITERATURE}

[34] SAP2000, Version 16.0.1, Computers and Structures Inc, 2013.

[35] CEB-FIP Model Code (1990), Thomas Telford Services Ltd., 1993 


\section{COMPUTER MODELLING OF STRUCTURES WITH ACCOUNT OF THE CONSTRUCTION STAGES AND THE TIME DEPENDENT MATERIAL PROPERTIES}

\section{Alexander TRAYKOV \\ Raina BOIADJIEVA}

Numerical studies are performed on computer models taking into account the stages of construction and time dependent material properties defined in two forms. A $2 D$ model of three storey two spans frame is created. The first form deals with material defined in the usual design practice way - without taking into account the time dependent properties of the concrete. The second form creep and shrinkage of the concrete are taken into account. Displacements and internal forces in specific elements and sections are reported. The influence of the time dependent material properties on the displacement and the internal forces in the main structural elements is tracked down.

The results corresponding to the two forms of material definition are compared together as well as with the results obtained by the usual design calculations. Conclusions on the influence of the concrete creep and shrinkage during the construction towards structural behaviour are made.

Key words: Computer model, 2D model, construction stages, creep and shrinkage of concrete

\section{NUMERIČKO MODELIRANJE KONSTRUKCIJA UZ UVOĐENJE FAZA GRAĐENJA I SVOJSTAVA MATERIJALA ZAVISNIH OD VREMENA}

\section{Alexander TRAYKOV \\ Raina BOIADJIEVA}

Numeričke analize na matematičkim modelima, kojima se uvode faze građenja i svojstva materijala zavisnih od vremena, definisane su u dva oblika. Model je dvodimenzionalni (2D) troetažni i s dva raspona. Prvi oblik tretira materijal definisan u uobičajenoj formi za projektantsku praksu - bez uvođenja svojstava betona zavisnih od vremena. U drugom obliku uvode se $u$ analizu skupljanje i tečenje betona. Prikazani su pomeranja $i$ presečne sile $u$ pojedinim elementima $i$ presecima. Izloženi su rezultati uticaja vremenski zavisnih svojstava materijala na pomeranja i presečne sile primarnih konstrukcijskih elemenata.

Rezultati odgovarajući za dva oblika definicije materijala upoređeni su $s$ rezultatima dobijenim uobičajenim proračunima pri projektovanju. Formulisani su odgovarajući zaključci o uticaju skupljanja i tečenja betona tokom izvođenja radova.

Ključne reči: proračunski model, 2D model, faze građenja, skupljanje i tečenje betona 